\title{
Preliminary open-label clinical evaluation of the soothing and reepithelialization properties of a novel topical formulation for rosacea
}

This article was published in the following Dove Press journal:

Clinical, Cosmetic and Investigational Dermatology

24 October 2014

Number of times this article has been viewed

\section{Adele Sparavigna \\ Beatrice Tenconi \\ Ileana De Ponti \\ Derming Srl, Monza, Italy}

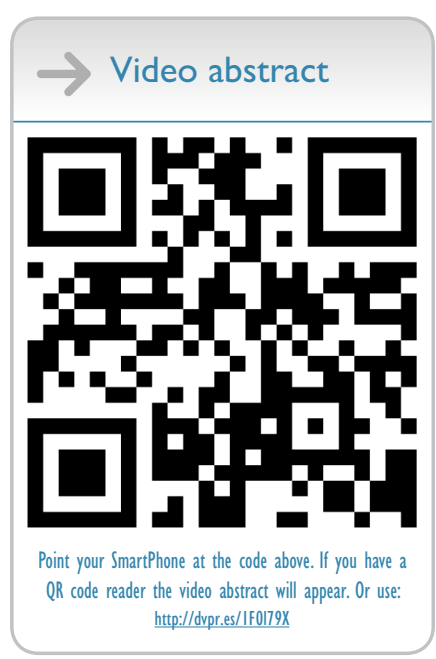

Correspondence: Adele Sparavigna Derming Srl, Clinical Research and Bioengineering Institute, Viale Cesare Battisti 38, Monza, Italy

Tel +3939329666

$\mathrm{Fax}+39392240210$

Email info@derming.com
Background: Rosacea is a common, incurable skin barrier disorder characterized by relapses and remissions.

Purpose: To evaluate the efficacy of Farmaka Rosacea Cream (FRC), a novel topical formulation for rosacea.

Methods: This single-center, open-label pilot study comprised a single-dose substudy in 20 healthy subjects and a long-term, repeat-dose substudy in 22 subjects with rosacea. The 2-hour, controlled, single-dose substudy assessed the soothing and reepithelialization properties of FRC after stripping-induced erythema based on the erythema index, transepidermal water loss, skin hydration, and clinical assessments of erythema. In the long-term substudy, subjects applied FRC twice daily for 8 weeks. Clinical assessments included vascular and pigmentary homogeneity and erythema and hemoglobin indices. Subjects completed questionnaires to assess FRC efficacy and cosmetic acceptability.

Results: Greater reductions were seen in FRC-treated areas compared with untreated areas for the erythema index $(-16 \%$ versus $-8 \% ; P<0.001)$ and mean transepidermal water loss $(-35.8 \%$ versus $-10.1 \% ; P<0.001) 30$ minutes after stripping. Significant improvements over untreated areas were maintained 2 hours after stripping. Skin hydration and clinical erythema assessments also indicated that FRC soothed rosacea symptoms and promoted skin reepithelialization. Erythema and hemoglobin indices were significantly reduced from baseline after 4 and 8 weeks of treatment. Clinically assessed parameters were significantly improved following FRC application. Subjects assessed FRC positively.

Conclusion: Improvement of rosacea symptoms was noted with FRC application. The main film-forming ingredients of FRC (trehalose, cholesterol, ceramide, and fatty acids), combined with other soothing and calming ingredients and ultraviolet filters, could explain its efficacy.

Keywords: rosacea, erythema, skin hydration, re-epithelialization

\section{Introduction}

Rosacea is a chronic inflammatory skin disorder characterized by relapses and remissions. Predominantly affecting the face, rosacea often begins with flushing and progresses to persistent redness and the appearance of visible blood vessels. If untreated, bumps and pimples develop and, in severe cases, the nose becomes swollen and bumpy (rhinophyma). Four clinical subtypes, with different symptoms, have been characterized (subtypes I-IV). ${ }^{1}$

With a reported prevalence varying from $<1 \%$ to $>20 \%$ in US and European populations, ${ }^{2}$ rosacea is more common in those aged $>30$ years, in fair-skinned people of Northern European descent, ${ }^{3}$ and in women. ${ }^{4}$ The causes of rosacea are complex and multifactorial, ${ }^{5}$ but involve damage to the skin's upper layer, the stratum corneum 
(SC), making it more susceptible to insult. Certain foods and environmental, chemical, and psychological factors aggravate rosacea, with ultraviolet radiation considered an important trigger. ${ }^{6}$ An elevated immune response and neurovascular dysregulation leads to heightened vasodilatory skin responses to various stimuli, ${ }^{7}$ and increased transepidermal water loss (TEWL), resulting from disruption of the SC permeability barrier, may contribute to skin dryness and sensitivity. ${ }^{8,9}$

Currently available treatments have limited efficacy and many can only be used for a short time. Topical prescription therapies include metronidazole, azelaic acid, and sulfacetamide-sulfur formulations. Rosacea can also be treated with oral antibiotics. In recalcitrant cases where antibiotics have failed, topical or oral treatment with retinoids may be indicated. ${ }^{10}$ Several reports have found lightbased treatments to be effective in reducing erythema and telangiectasia, including long-pulsed dye, potassium titanyl phosphate, and diode lasers, which have been associated with little or no purpura. They also may be reduced by intense pulsed light therapy and electrocautery. ${ }^{11}$ In addition, basic skincare regimens, including the daily use of a sunscreen, moisturizers, and appropriate nonirritating cosmetics and non-soap cleanser, may offer significant benefits - enhancing skin properties and relieving skin discomfort. ${ }^{12}$

Farmaka Rosacea Cream (FRC; Farmaka srl, Milan, Italy) is a novel topical formulation containing a patented combination of ingredients that form a protective film on the skin. The combination of trehalose and key skin elements (cholesterol, ceramide, and fatty acids) moisturize the skin, restoring and hydrating the upper layer. Soothing and calming ingredients (Echinacea angustifolia extract, bisabolol, esculin, Boswellia serrata resin extract) and specific ultraviolet filters (ethylhexyl methoxycinnamate [PARSOL ${ }^{\circledR}$ MCX], butyl methoxydibenzoylmethane [PARSOL ${ }^{\circledR} 1789$ ]) may also be effective against the main symptoms and triggers of rosacea. This pilot study evaluated the soothing and reepithelialization activity of a single application of FRC on experimentally induced erythema, as well as the anti-couperose efficacy of FRC after repeated application. Tolerability, antiaging/photoaging properties, and cosmetic acceptability were also assessed.

\section{Materials and methods Study design}

This single-center, open-label study, conducted by Derming Srl (Monza, Italy), consisted of a short-term controlled substudy in healthy subjects and a long-term substudy in subjects with rosacea. Data were generated, recorded, and processed in accordance with the International Conference on Harmonization of Technical Requirements for Registration of Pharmaceuticals for Human Use (ICH) guidelines for good clinical practice. ${ }^{13}$ Informed consent was provided by all subjects prior to study entry. Since 2011, FRC has been launched in several European Union countries and has therefore been used extensively by subjects with rosacea following this study. The full protocol for this trial is available at Derming Srl.

\section{Subjects}

Healthy female subjects aged $>18$ years, who agreed not to receive treatment that would change their skin characteristics for the study duration or to use anti-rosacea or -couperose products for a month preceding the study, were recruited. Subjects in the long-term substudy had rosacea subtype I (erythematotelangiectatic; redness, flushing, and visible blood vessels) ${ }^{1}$ or subtype II (papulopustular; redness, swelling, papules/pustules, and acne-like breakouts). ${ }^{1}$

For both substudies, subjects with dermatitis or eczema were excluded, as were subjects with diabetes, endocrine disease, hepatic, renal or cardiac disorders, pulmonary disease, cancer, neurological or psychological disease, inflammatory/ immunosuppressive disease, or drug allergy. Pregnant or lactating women, subjects who changed their normal lifestyle in the month preceding the investigation, and those with hypersensitivity to the product or its ingredients were also excluded. Subjects with recurrent facial/labial herpes were excluded from the long-term substudy.

\section{Study treatment}

For the short-term substudy, skin erythema was induced by positioning a freshly cyanoacrylate-coated microscope slide onto the skin of subjects' backs for approximately 60 seconds. This "skin stripping" was performed three consecutive times on each control and test area. Immediately after stripping, FRC $\left(2.00 \mathrm{mg} / \mathrm{cm}^{2}\right)$ was applied to the test area by the investigator using light massage and left to absorb. The control area was left untreated.

Subjects in the long-term substudy applied FRC at home twice daily (morning and evening) on the face for 8 weeks, using light massage, until complete absorption.

In both substudies, subjects were randomly assigned in a 1:1 ratio to receive FRC on the left or right side, according to a previously generated randomization list. Use of systemic corticosteroids, aspirin, or nonsteroidal anti-inflammatory drugs, antihistamines, narcotics, 
antidepressants, immunosuppressive drugs, or drugs that could influence the test results was prohibited.

\section{Study assessments and endpoints}

Subjects could not smoke, drink coffee or alcohol, or use any product on the face for 3 hours before the visit. All measurements were performed under standard environmental conditions (temperature $22^{\circ} \mathrm{C} \pm 2{ }^{\circ} \mathrm{C}$; relative humidity $\leq 60 \%$ ), and subjects were acclimatized under relaxed conditions for at least 15 minutes prior to testing. Adverse or serious adverse events were recorded. In both substudies, the erythema index (EI) was evaluated using an optical densitometer (X-Rite ${ }^{\circledR}$ model 404; X-Rite, Inc., Grand Rapids, MI, USA) and was calculated as follows:

$$
\mathrm{EI}=\log \mathrm{R}_{\text {magenta }}-\log \mathrm{R}_{\text {cyan }}
$$

\section{Short-term substudy}

Evaluations were performed bilaterally on treated and untreated areas at baseline $\left(\mathrm{T}_{0}\right.$; before product application), immediately after stripping $\left(\mathrm{T}_{\mathrm{i}}\right)$, and 30 minutes $\left(\mathrm{T}_{30 \mathrm{~m}}\right)$ and 2 hours $\left(\mathrm{T}_{2 \mathrm{~h}}\right)$ after a single application of FRC. Erythema was scored by a dermatologist according to a five-point scale, with zero being "no erythema" and four being "severe erythema."

Skin barrier function (TEWL) was assessed using an Evaporimeter EP2 (Servo Med AB, Kinna, Sweden). Electrical skin capacitance (hydration) was measured using a Corneometer ${ }^{\circledR}$ CM820 (Courage + Khazaka electronic $\mathrm{GmbH}$, Cologne, Germany).

\section{Long-term substudy}

Evaluations were performed unilaterally on the face at baseline $\left(\mathrm{T}_{0}\right.$; before product application), at Week $4\left(\mathrm{~T}_{4}\right)$ and at the final Week 8 visit $\left(\mathrm{T}_{8}\right)$. Instrumental evaluations were performed at least 8 hours after the last application of FRC.

Wrinkles (at the nasolabial folds and area around the eyes), surface microrelief, skin tone/elasticity, skin brightness, dryness, and vascular and pigmentary homogeneity were scored according to visual scales. Facial clinical signs (excluding skin brightness) were correlated using a proprietary “spider web" graph (Spiderming $\left.{ }^{\circledR}\right),{ }^{14}$ which provides a fully comprehensive evaluation of aging skin.

Hemoglobin spectroscopic evaluation was performed using a SIAscope ${ }^{\mathrm{TM}}$ (Astron Clinica, Cambridge, UK), which visualizes skin structure, vascular composition, and reticular pigment networks and measures the concentration and distribution of skin melanin, collagen, and hemoglobin.
Photographic recovery was performed using the FotoFinder ${ }^{\circledR}$ Dermoscope (FotoFinder Systems GmbH, Bad Birnbach, Germany) with a magnification of $20 \times$.

At $\mathrm{T}_{8}$, the investigator interviewed each subject to determine the cosmetic acceptability of treatment. The tolerance and efficacy of FRC was evaluated using a subject questionnaire and investigator assessments, including any treatment-related adverse events or events that may have interfered with the results. At $\mathrm{T}_{4}$ and $\mathrm{T}_{8}$, compliance with the treatment regimen was verified by evaluation of personal diary cards in which subjects recorded the date and time of each FRC application.

\section{Statistical analysis}

Statistical analysis of short-term efficacy was performed for $\mathrm{T}_{0}$ and $\mathrm{T}_{\mathrm{i}}$ using the Friedmann test for clinical evaluations and analysis of variance for instrumental evaluations. If the initial test was statistically significant, Tukey's post hoc analysis was used. Comparisons of the treated and untreated (control) skin patches at $\mathrm{T}_{30 \mathrm{~m}}$ and $\mathrm{T}_{2 \mathrm{~h}}$ were performed using the Wilcoxon test for clinical evaluations and Student's $t$-test for instrumental evaluations.

Long-term evaluation of efficacy at $T_{4}$ and $T_{8}$ versus $T_{0}$ was performed using the Friedmann test for clinical evaluations and analysis of variance for instrumental evaluations. If the initial test was statistically significant, Dunnett's post hoc analysis was used. Although no sample size calculations were performed, previous studies of the efficacy of treatments for rosacea have used similar numbers of patients. ${ }^{15,16}$

\section{Results \\ Subjects}

Forty-two of the 48 recruited subjects received treatment. In the short-term substudy, 20 subjects, with a mean age of 48 years (range 23-69 years), were treated. The long-term population comprised 22 subjects with rosacea (ten subjects [45\%] subtype I; 12 subjects [55\%] subtype II), with a mean age of 56 years (range 32-69 years). Half (50\%) of the subjects in each substudy received treatment on the left side and half $(50 \%)$ on the right side. In the long-term substudy, skin was classified as dry (eleven subjects [50\%]), combination (six subjects [27\%]), or normal (five subjects [23\%]), with a skin phototype (Cesarini's classification) of subtype II or IV (nine subjects each [41\%]), subtype III (three subjects [13\%]), or subtype V (one subject [5\%]). Two subjects from the long-term population discontinued due to personal reasons unrelated to FRC. Thus, 20 subjects completed the long-term substudy (Figure 1). 


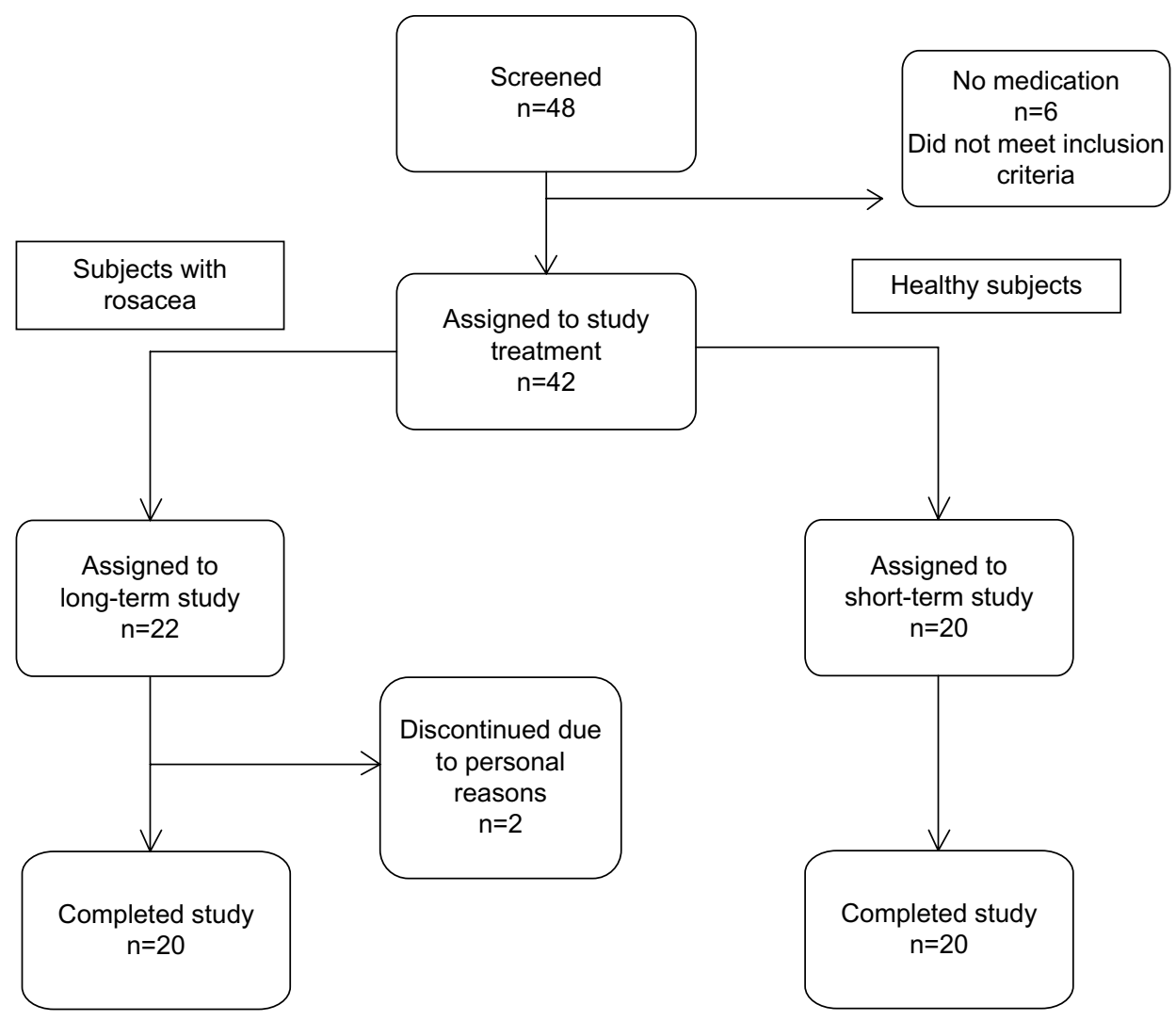

Figure I Study flow diagram.

\section{Short-term evaluation of efficacy}

Investigator assessments revealed no significant difference in erythema intensity between control and treated areas at $T_{i}$, confirming that skin stripping consistently induced erythema. At $\mathrm{T}_{30 \mathrm{~m}}$, a statistically significant reduction in skin erythema was observed on FRC-treated skin $\left(-43.8 \%\right.$ at $\mathrm{T}_{30 \mathrm{~m}}$ versus $\mathrm{T}_{0} ; P<0.05$ ), although the reduction was not significantly different to that of untreated skin $(-27.1 \%)$. At $\mathrm{T}_{2 \mathrm{~h}}$, however, erythema was reduced by a significantly greater extent on FRC-treated skin $(-87.2 \%)$ compared with the control $(-73.6 \% ; P<0.01)$.

Skin stripping caused a statistically significant increase of the EI compared with $\mathrm{T}_{0}$ on treated and untreated areas (38.9\% increase in both; $P<0.05)$. After FRC application, there was a statistically significant reduction of the EI compared with the control (untreated) area at $\mathrm{T}_{30 \mathrm{~m}}(-16 \%$ versus $-8 \% ; P<0.001)$ and $\mathrm{T}_{2 \mathrm{~h}}(-28 \%$ versus $-20 \% ; P<0.01)$ (Figure 2).

A significant increase in TEWL compared with baseline $(P<0.05)$ was observed at $\mathrm{T}_{\mathrm{i}}$ for treated and untreated areas, suggesting the cutaneous barrier was damaged by skin stripping. After FRC application, TEWL was reduced by $35.8 \%$ at $\mathrm{T}_{30 \mathrm{~m}}$ compared with $\mathrm{T}_{\mathrm{i}}(P<0.05)$, returning to a level comparable to baseline $\left(\mathrm{T}_{30 \mathrm{~m}} 5.25 \mathrm{~g} / \mathrm{m}^{2} /\right.$ hour versus $\mathrm{T}_{0} 4.74 \mathrm{~g} / \mathrm{m}^{2} /$ hour). This reduction was significantly greater than the reduction observed in the control area $(-35.8 \%$ versus $-10.1 \% ; P<0.001)$. At $\mathrm{T}_{2 \mathrm{~h}}$, TEWL remained low with no statistical difference from $\mathrm{T}_{0}$, whereas control areas had sustained barrier dysfunction (Figure 3).

At $T_{i}$, a statistically significant increase in electrical capacitance (hydration) was reported for the control skin compared with $\mathrm{T}_{0}(P<0.05)$, whereas the increase in areas to be treated with FRC was not significant. At $\mathrm{T}_{30 \mathrm{~m}}$ and $\mathrm{T}_{2 \mathrm{~h}}$, statistically significant differences in capacitance were reported between treated and untreated skin $(P<0.001)$. For untreated skin, capacitance at both time points remained similar to those at $\mathrm{T}_{\mathrm{i}}\left(73.3\right.$ at $\mathrm{T}_{\mathrm{i}}, 75.3$ at $\mathrm{T}_{30 \mathrm{~m}}$, and 75.0 at $\mathrm{T}_{2 \mathrm{~h}}$ ). In FRC-treated skin, statistically significant increases in capacitance were observed at $\mathrm{T}_{30 \mathrm{~m}}$ and $\mathrm{T}_{2 \mathrm{~h}}$ compared with $\mathrm{T}_{\mathrm{i}}\left(74.3\right.$ at $\mathrm{T}_{\mathrm{i}}, 87.2$ at $\mathrm{T}_{30 \mathrm{~m}}$, and 81.9 at $\mathrm{T}_{2 \mathrm{~h}} ; P<0.05$ ).

\section{Long-term evaluation of efficacy}

Compared with $\mathrm{T}_{0}$, statistically significant reductions were reported for EI $(-18 \% ; P<0.05)$ and skin hemoglobin $(-21 \% ; P<0.05)$ at $\mathrm{T}_{4}$, which were maintained for the 8 week treatment period (EI $-27 \%$ and hemoglobin $-34 \%$ 


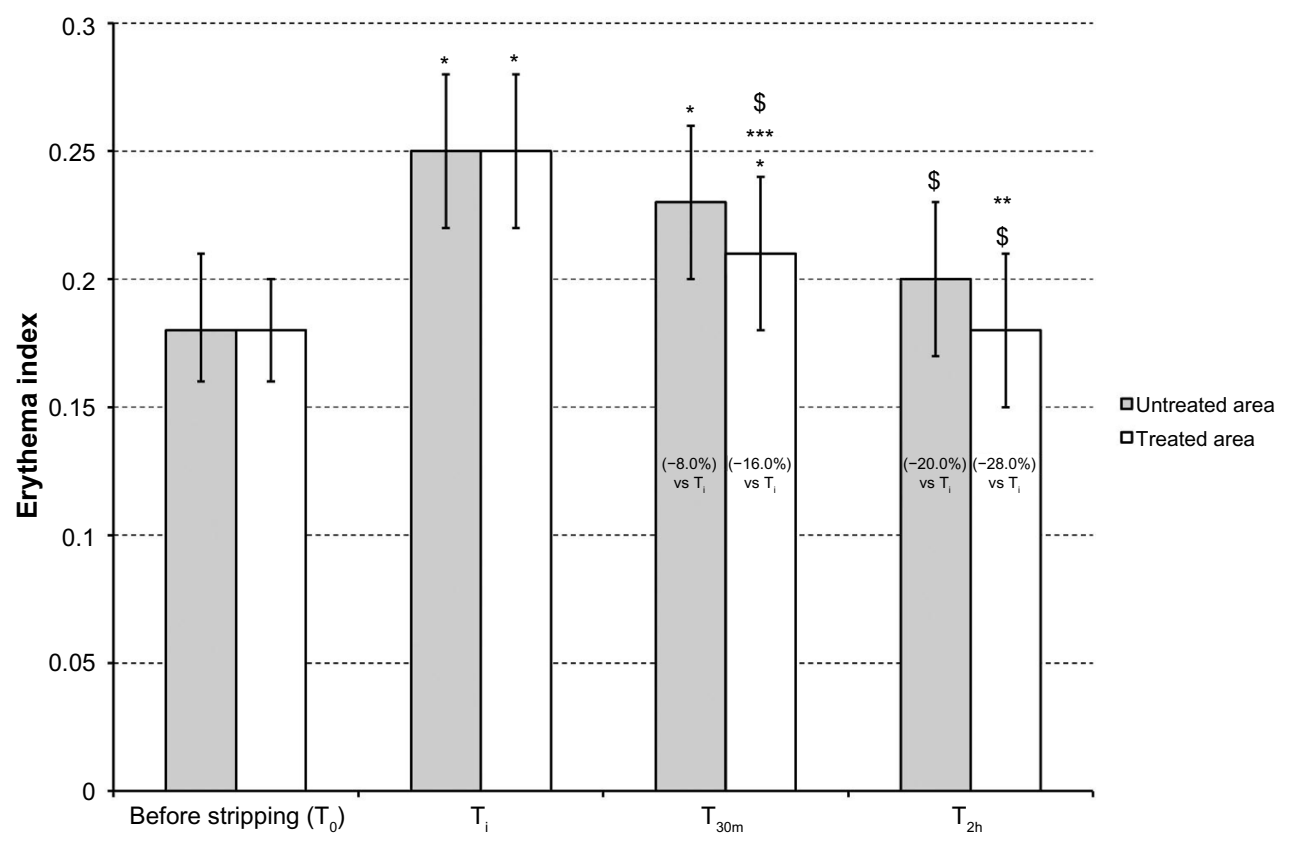

Figure 2 Farmaka Rosacea Cream reduces stripping-induced erythema. Mean erythema index ( \pm 0.5 standard deviations) before stripping ( $\left.\mathrm{T}_{0}\right)$, immediately after stripping $\left(T_{i}\right), 30$ minutes after stripping $\left(T_{30 m}\right)$, and 2 hours after stripping $\left(T_{2 h}\right)$.

Notes: $* P<0.05 T_{i}$ and $T_{30 m}$ versus $T_{0} ; * * P<0.01$ treated versus untreated at $T_{2 h}$; **** $<<0.001$ treated versus untreated at $T_{30 m} ; \$ p<0.05 T_{30 m}$ and $T_{2 h}$ versus $T_{i}$. The numbers in brackets denote the percentage decrease in the erythema index compared with $T_{i}$.

at $\mathrm{T}_{8}$ versus $\mathrm{T}_{0} ; P<0.05$ ) (Figure 4 ). A statistically significant reduction in vascular homogeneity score was observed after 4 weeks $\left(-55 \%\right.$ at $\mathrm{T}_{4}$ versus $\left.\mathrm{T}_{0} ; P<0.05\right)$ and 8 weeks $\left(-75 \%\right.$ at $\mathrm{T}_{8}$ versus $\left.\mathrm{T}_{0} ; P<0.05\right)$ of treatment. Video dermoscopy revealed a reduction in capillary diameter (Figure 5).
The "spider web" graph (Figure 6) revealed statistically significant improvements in vascular and pigmentary homogeneity at $\mathrm{T}_{4}$ and $\mathrm{T}_{8}$. Furthermore, $60 \%$ and $70 \%$ of the subjects demonstrated an improvement in pigmentary homogeneity at $\mathrm{T}_{4}$ and $\mathrm{T}_{8}$, respectively, compared with $\mathrm{T}_{0}$ $(P<0.05)$. All other parameters represented on the graph

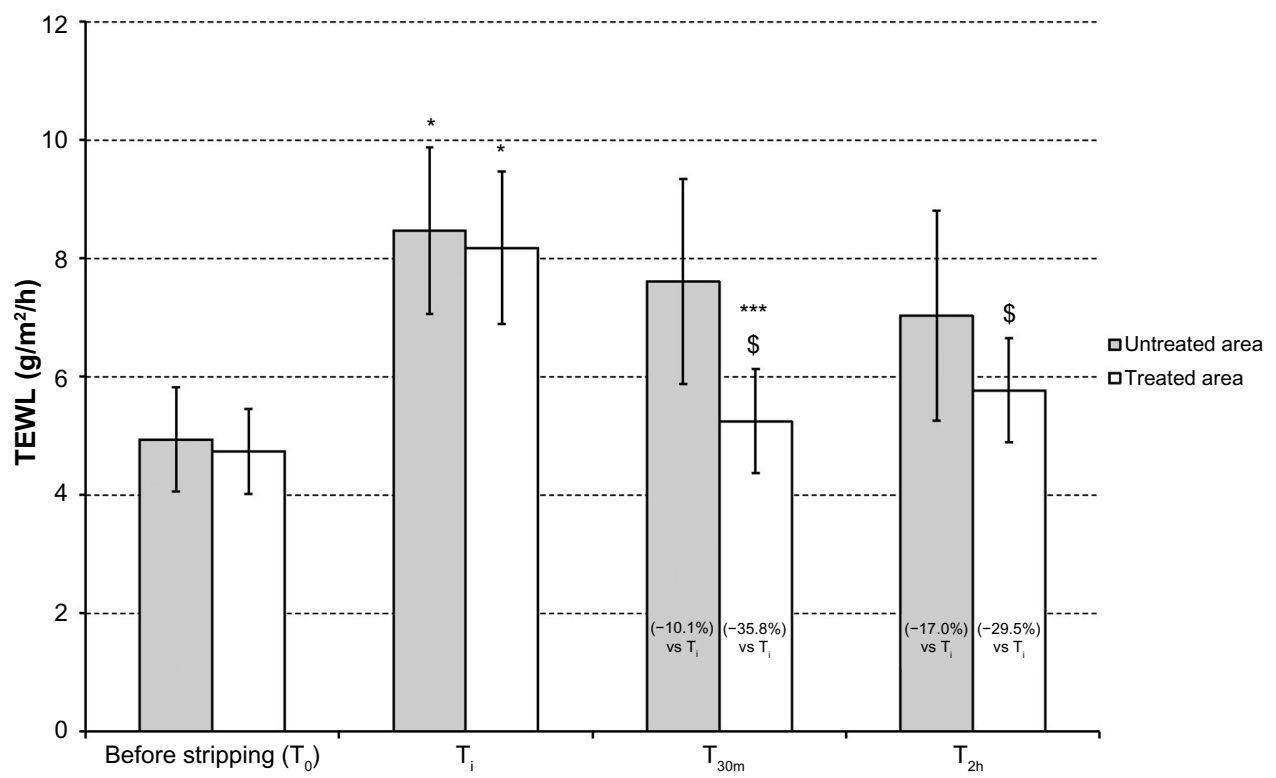

Figure 3 Farmaka Rosacea Cream reduces transepidermal water loss (TEWL). Mean TEWL ( \pm 0.5 standard deviations) before stripping $\left(T_{0}\right)$, immediately after stripping $\left(T_{i}\right)$, 30 minutes after stripping $\left(T_{30 \mathrm{~m}}\right)$, and 2 hours after stripping $\left(\mathrm{T}_{2 \mathrm{~h}}\right)$.

Notes: $* P<0.05 \mathrm{~T}_{i}$ versus $\mathrm{T}_{0} ; * * * P<0.001$ treated versus untreated at $\mathrm{T}_{30 \mathrm{~m}} ;{ }^{\$ P}<0.05 \mathrm{~T}_{30 \mathrm{~m}}$ and $\mathrm{T}_{2 \mathrm{~h}}$ versus $\mathrm{T}_{\mathrm{i}}$. The numbers in brackets denote the percentage decrease in TEWL compared with $T_{i}$. 


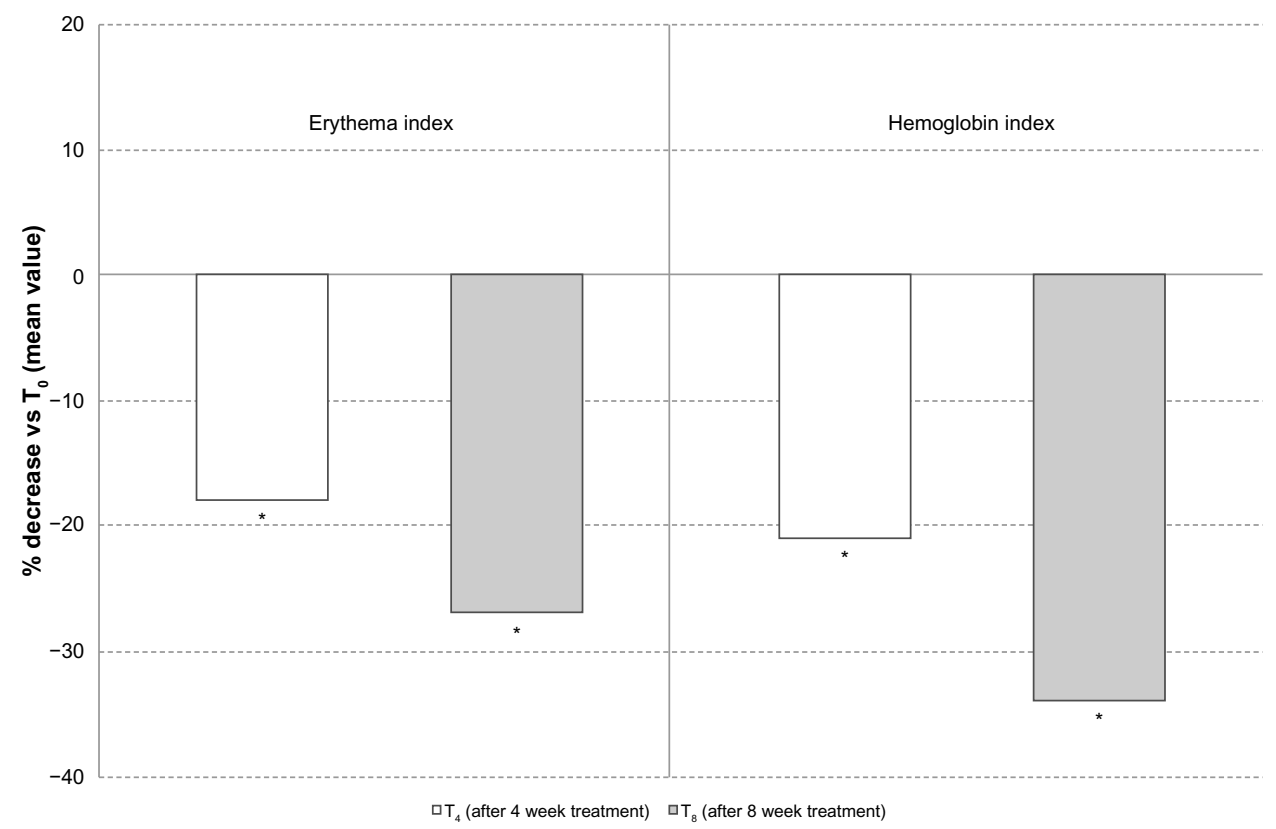

Figure 4 Farmaka Rosacea Cream decreases both erythema and cutaneous hemoglobin indices. Mean erythema and hemoglobin index ( \pm 0.5 standard deviations) at baseline $\left(T_{0}\right)$, after 4 weeks of treatment $\left(T_{4}\right)$, and after 8 weeks of treatment $\left(T_{8}\right)$.

Note: $* P<0.05$.

Example 3
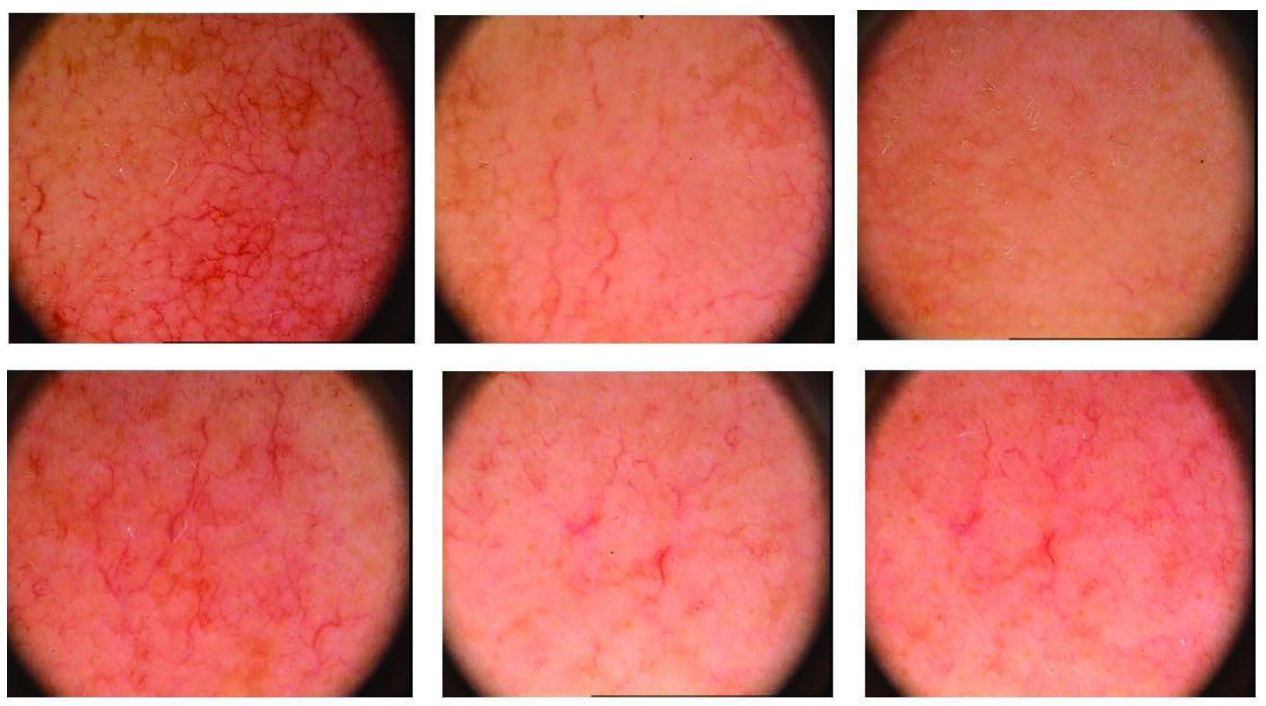

Example 2
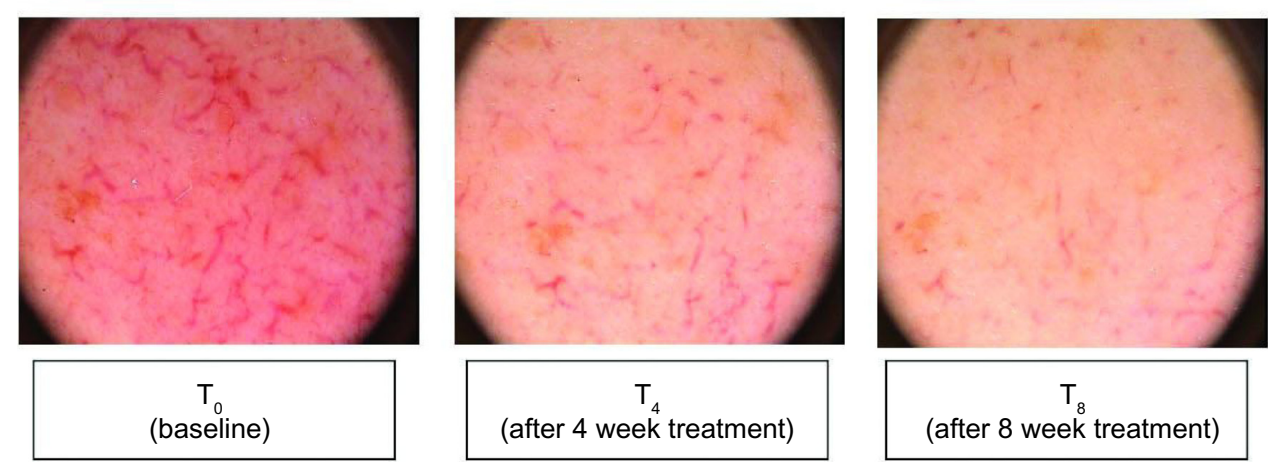

Figure 5 Farmaka Rosacea Cream reduces capillary caliber after 4 weeks $\left(T_{4}\right)$ and 8 weeks $\left(T_{8}\right)$ of treatment compared with baseline $\left(T_{0}\right)$. 


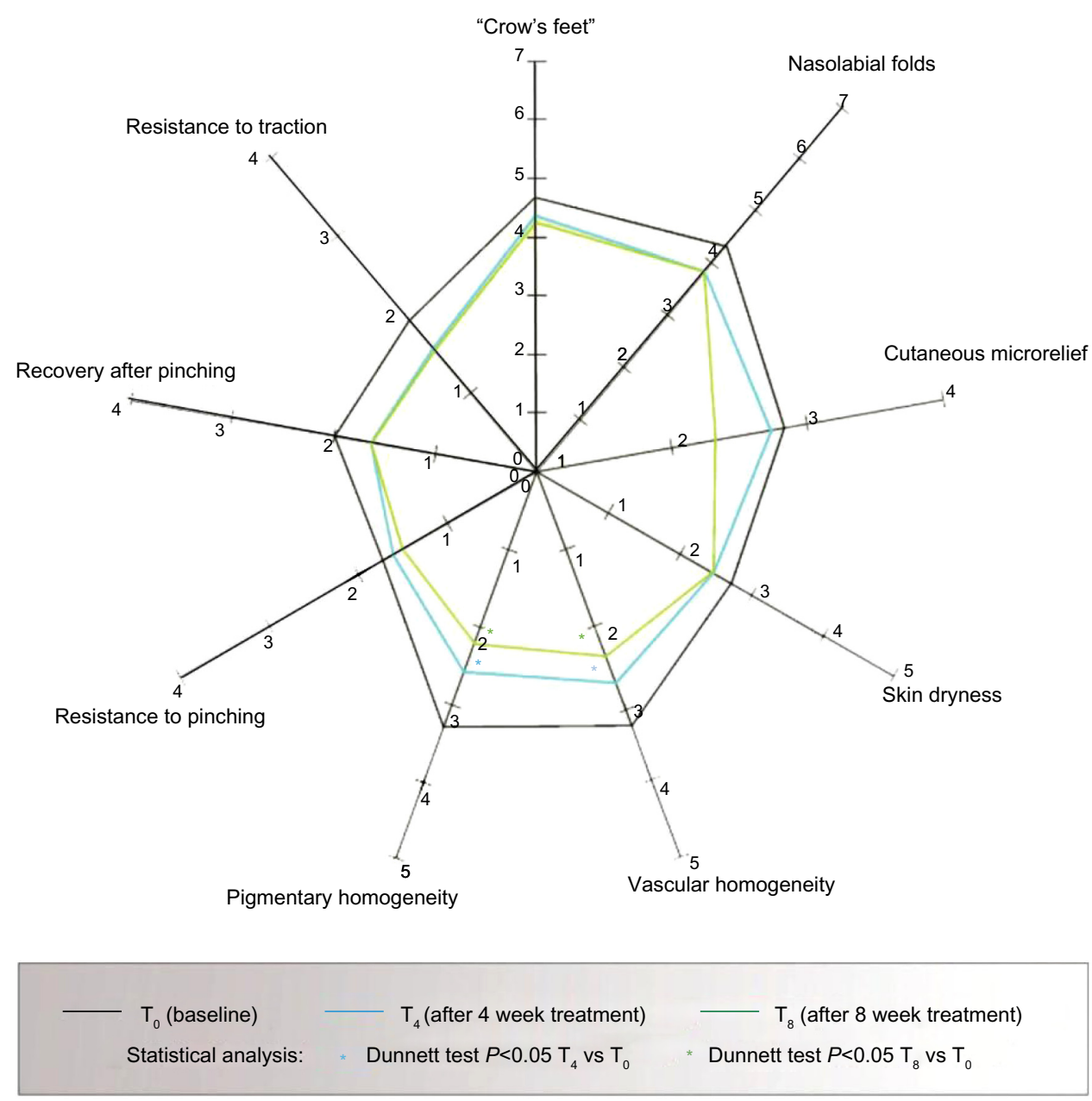

Figure 6 Farmaka Rosacea Cream improves vascular and pigmentary homogeneity. The mean values of the tested parameters were depicted using a "spider web" graph (Spiderming ${ }^{8}$ ). After 4 weeks of treatment $\left(T_{4}\right), 60 \%$ of subjects demonstrated an improvement of vascular homogeneity, while after 8 weeks of treatment $\left(T_{8}\right)$, a statistically significant improvement of pigmentary homogeneity was measured in $70 \%$ of the subjects $\left(P<0.05 \mathrm{~T}_{0}\right.$ versus $\mathrm{T}_{4}$ and $\left.\mathrm{T}_{8}\right)$.

improved after treatment with FRC but did not reach statistical significance.

A high proportion of subjects $(60 \%-100 \%)$ judged the effect of FRC to be medium, marked, or very marked for all efficacy parameters. Generally, the cosmetic acceptability of FRC was rated as good. The tolerability of FRC was viewed as good or excellent by $100 \%$ of subjects and investigators, with no adverse effects considered related or unrelated to the product.

\section{Discussion}

Rosacea is thought to be triggered by damage to the skin's natural barrier (the SC) and increasing evidence indicates a role of sebaceous fatty acids in the maintenance of SC acidification and integrity. ${ }^{17}$ The combination of ingredients in FRC aims to reduce flare-ups, protect the skin from the main triggers of rosacea, and soothe and calm the skin. This study investigated both short- and long-term use of FRC.

After stripping-induced skin damage in subjects without rosacea, FRC elicited statistically significant improvements in the skin's EI (soothing effect), barrier functionality (measured by TEWL), and hydration (measured by electrical capacitance), which occurred as soon as 30 minutes after application and were maintained at 2 hours posttreatment. In the long-term substudy, application of FRC twice daily for 8 weeks also resulted in statistically significant improvements in rosacea signs and symptoms, demonstrated by significant decreases in erythema and hemoglobin indices after 4 weeks of treatment. These reductions were maintained or increased after 8 weeks, suggesting that regular and prolonged treatment may be viable. At the end of the study, skin color was more homogeneous, with visible improvements in skin aging signs such as dryness and crow's feet. 
These preliminary results indicate a potential clinical benefit for patients with rosacea. However, although the efficacy of FRC compared to untreated areas was demonstrated in the short-term substudy, a control group was not included in the long-term substudy. The effects of FRC over placebo would be of interest, as repeated application of cream to the affected skin may have had a nonspecific soothing effect. These preliminary studies were also not blinded and while a number of objective, instrumental assessments were employed, several also included a subjective component. Nonetheless, these initial results suggest a beneficial effect of FRC for relief of rosacea symptoms and indicate that further blinded, placebo-controlled, and/or split-face assessment of FRC is warranted. Additionally, although rosacea most frequently occurs in females aged $>30$ years ${ }^{3,4}$ and the study population was representative of this group, assessment of FRC in men would be of interest.

Previous studies of rosacea treatments have predominantly focused on the efficacy of oral antibiotics (eg, doxycycline) or topical metronidazole and azelaic acid. ${ }^{1,18-22}$ However, unlike the current study, none assessed the subjects' views and satisfaction with treatment. ${ }^{23}$ Importantly, most subjects and investigators regarded FRC's efficacy and tolerability to be good or excellent in the current study.

To the best of the authors' knowledge, no published clinical trials have directly studied the efficacy of FRC constituents on rosacea symptoms. However, data from a number of studies provide support for the contribution of individual constituents to the efficacy observed. De Paepe et $\mathrm{al}^{24}$ reported that lotioned handkerchiefs containing fatty alcohols and mineral oils helped prevent skin damage caused by the wiping on skin-stripped forearms of volunteers. Additionally, topical application of a combination nanoparticle product (trehalose, ceramide, and cholesterol) increased skin capacitance by up to $40 \%$ and decreased TEWL by $15 \%$ compared with placebo. ${ }^{25}$ These data suggest that the fatty acid and sugar components of FRC contribute to its efficacy in reducing the signs and symptoms of rosacea.

Other components of FRC include ceramides and fatty acids, which a number of studies have shown to be involved in maintenance of the SC. Ceramides represent the simplest sphingolipids, ${ }^{26}$ which are major, essential components of the epidermal water barrier. Inhibition of sphingolipid synthesis is accompanied by a significant delay in the rate of barrier recovery after acute disruption in murine epidermis. ${ }^{27}$ FRC also contains cholesterol, an extracellular and intracellular fatty acid and the main constituent of the cellular membrane. ${ }^{28}$ Inhibition of epidermal fatty acid synthesis in stripped skin significantly delayed barrier recovery in male hairless mice, while providing exogenous fatty acids by co-application of palmitate was found to improve barrier recovery. ${ }^{29}$ Topical provision of missing lipids, either cholesterol or sphingolipid, can also overcome inhibition of skin barrier repair. ${ }^{27,30,31}$ Together, the results of these studies have confirmed the significance of lipids for the construction and repair of the SC, which could partly explain the efficacy of FRC.

A key component of FRC is the naturally occurring disaccharide trehalose, which is widely used in the cosmetics, medical device, and pharmaceutical industries for its moisturizing properties. ${ }^{32}$

\section{Conclusion}

Short-term application of FRC reduced erythema in healthy subjects following skin stripping, while longer-term treatment in subjects with rosacea helped to counter the associated signs and symptoms. The fatty acid and sugar content of FRC could explain its efficacy in the treatment of rosacea.

\section{Acknowledgments}

This study was funded by Farmaka srl (Milan, Italy). The authors thank Insight Medical Writing (Kidlington, UK) for assistance in the preparation of this manuscript.

\section{Disclosure}

The study was funded by the manufacturer of the rosacea cream. The authors report no other conflicts of interest in this work.

\section{References}

1. Goldgar C, Keahey DJ, Houchins J. Treatment options for acne rosacea. Am Fam Physician. 2009;80(5):461-468.

2. Chosidow O, Cribier B. Epidemiology of rosacea: updated data. Ann Dermatol Venereol. 2011;138(Suppl 3):S179-S183.

3. Blount BW, Pelletier AL. Rosacea: a common, yet commonly overlooked, condition. Am Fam Physician. 2002;66(3):435-440.

4. Berg M, Liden S. An epidemiological study of rosacea. Acta Derm Venereol. 1989;69(5):419-423.

5. Levin J, Miller R. A guide to the ingredients and potential benefits of over-the-counter cleansers and moisturizers for rosacea patients. J Clin Aesthet Dermatol. 2011;4(8):31-49.

6. Murphy G. Ultraviolet light and rosacea. Cutis. 2004;74(Suppl 3):13-16, 32-34.

7. Del Rosso JQ. Advances in understanding and managing rosacea: part 1: connecting the dots between pathophysiological mechanisms and common clinical features of rosacea with emphasis on vascular changes and facial erythema. J Clin Aesthet Dermatol. 2012;5(3):16-25.

8. Dirschka T, Tronnier H, Folster-Holst R. Epithelial barrier function and atopic diathesis in rosacea and perioral dermatitis. Br J Dermatol. 2004;150(6):1136-1141.

9. Lonne-Rahm SB, Fischer T, Berg M. Stinging and rosacea. Acta Derm Venereol. 1999;79(6):460-461.

10. Cohen AF, Tiemstra JD. Diagnosis and treatment of rosacea. $J$ Am Board Fam Pract. 2002;15(3):214-217. 
11. Odom R, Dahl M, Dover J, et al. Standard management options for rosacea, part 2: options according to subtype. Cutis. 2009;84(2): 97-104.

12. Culp B, Scheinfeld N. Rosacea: a review. P T. 2009;34(1):38-45.

13. International Conference on Harmonization of Technical Requirements for Registration of Pharmaceuticals for Human Use. ICH harmonized tripartite guideline: guideline for good clinical practice. J Postgrad Med. 2001;47(1):45-50.

14. Serri R, Romano MC, Sparavigna A. "Quitting smoking rejuvenates the skin": results of a pilot project on smoking cessation conducted in Milan, Italy. Skinmed. 2010;8(1):23-29.

15. Tremaine AM, Ortiz A, Elkeeb L, Tran M, Weinstein G. Long-term efficacy and safety of topical PRK $124(0.125 \%)$ lotion (Pyratine-XR) in the treatment of mild-to-moderate rosacea. J Drugs Dermatol. 2010;9(6):647-650.

16. Leyden JJ. Efficacy of a novel rosacea treatment system: an investigator-blind, randomized, parallel-group study. J Drugs Dermatol. 2011;10(10):1179-1185.

17. Fluhr JW, Kao J, Jain M, Ahn SK, Feingold KR, Elias PM. Generation of free fatty acids from phospholipids regulates stratum corneum acidification and integrity. J Invest Dermatol. 2001;117(1): 44-51.

18. Del Rosso JQ, Webster GF, Jackson M, et al. Two randomized Phase III clinical trials evaluating anti-inflammatory dose doxycycline (40-mg doxycycline, USP capsules) administered once daily for treatment of rosacea. J Am Acad Dermatol. 2007;56(5):791-802.

19. Fowler JF Jr. Combined effect of anti-inflammatory dose doxycycline (40-mg doxycycline, USP monohydrate controlled-release capsules) and metronidazole topical gel $1 \%$ in the treatment of rosacea. J Drugs Dermatol. 2007;6(6):641-645.

20. Sanchez J, Somolinos AL, Almodovar PI, Webster G, Bradshaw M, Powala C. A randomized, double-blind, placebo-controlled trial of the combined effect of doxycycline hyclate $20-\mathrm{mg}$ tablets and metronidazole $0.75 \%$ topical lotion in the treatment of rosacea. $J$ Am Acad Dermatol. 2005;53(5):791-797.

21. Elewski BE, Fleischer AB Jr, Pariser DM. A comparison of $15 \%$ azelaic acid gel and $0.75 \%$ metronidazole gel in the topical treatment of papulopustular rosacea: results of a randomized trial. Arch Dermatol. 2003;139(11):1444-1450.
22. Maddin S. A comparison of topical azelaic acid $20 \%$ cream and topical metronidazole $0.75 \%$ cream in the treatment of patients with papulopustular rosacea. J Am Acad Dermatol. 1999; 40(6 Pt 1):961-965.

23. van Zuuren EJ, Kramer S, Carter B, Graber MA, Fedorowicz Z. Interventions for rosacea [review]. Cochrane Database Syst Rev. 2011;3:CD003262.

24. De Paepe K, De Rop E, Houben E, Adam R, Rogiers V. Effects of lotioned disposable handkerchiefs on skin barrier recovery after tape stripping. Skin Res Technol. 2008;14(4):440-447.

25. Sinerga Spa. Tri-Solve: High Performance Modulator for Skin Barrier Recovery. Pero: Sinerga; 2008. Available from: http:/www.sinerga.it/ news/VOLANTINO\%20TRI-SOLVE.pdf. Accessed September 15, 2014.

26. Schreiner V, Gooris GS, Pfeiffer S, et al. Barrier characteristics of different human skin types investigated with X-ray diffraction, lipid analysis, and electron microscopy imaging. J Invest Dermatol. 2000;114(4): 654-660.

27. Holleran WM, Man MQ, Gao WN, Menon GK, Elias PM, Feingold KR. Sphingolipids are required for mammalian epidermal barrier function. Inhibition of sphingolipid synthesis delays barrier recovery after acute perturbation. J Clin Invest. 1991;88(4):1338-1345.

28. Sinerga SpA. Tri-Solve: high performance skin barrier recovery. Pero: Sinerga SpA; 2009. Available from: http://www.sinerga.it/schede/ st_en_tri-solve.pdf. Accessed October 6, 2014.

29. Mao-Qiang M, Elias PM, Feingold KR. Fatty acids are required for epidermal permeability barrier function. J Clin Invest. 1993;92(2):791-798.

30. Feingold KR, Man MQ, Menon GK, Cho SS, Brown BE, Elias PM Cholesterol synthesis is required for cutaneous barrier function in mice. J Clin Invest. 1990;86(5):1738-1745.

31. Menon GK, Feingold KR, Mao-Qiang M, Schaude M, Elias PM. Structural basis for the barrier abnormality following inhibition of $\mathrm{HMG}$ CoA reductase in murine epidermis. J Invest Dermatol. 1992;98(2): 209-219.

32. Teramoto N, Sachinvala ND, Shibata M. Trehalose and trehalose-based polymers for environmentally benign, biocompatible, and bioactive materials. Molecules. 2008;13(8):1773-1816.
Clinical, Cosmetic and Investigational Dermatology

\section{Publish your work in this journal}

Clinical, Cosmetic and Investigational Dermatology is an international, peer-reviewed, open access, online journal that focuses on the latest clinical and experimental research in all aspects of skin disease and cosmetic interventions. All areas of dermatology will be covered; contributions will be welcomed from all clinicians and

\section{Dovepress}

basic science researchers globally. This journal is indexed on CAS. The manuscript management system is completely online and includes a very quick and fair peer-review system, which is all easy to use. Visit http://www.dovepress.com/testimonials.php to read real quotes from published authors. 